\title{
Fewer Lasers Achieve Higher Fidelity Logic Gate
}

\author{
Researchers halve the number of lasers needed to implement a quantum \\ logic gate between two different atomic species, a feat that could help to \\ create a scalable quantum computer. \\ By Katherine Wright
}

\rceil

he race is on to build the first large-scale quantum computer. A plethora of routes exist for doing that, including one adopted by Amy Hughes, Vera Schäfer, Keshav Thirumalai, and their colleagues at the University of Oxford, UK, that would link ionic qubits together using optical fibers [1]. Such a computer requires ions for processing, storing, and transferring information. No single ion species has the perfect properties needed for all these tasks, so the team-like others taking this route-plans to combine two different species. In new experiments they demonstrate the transfer of quantum information between two different ions, ${ }^{43} \mathrm{Ca}+$ and ${ }^{88} \mathrm{Sr}^{+}$, with fewer errors than in previous experiments.

In their setup, the team first trapped individual ${ }^{43} \mathrm{Ca}^{+}$and ${ }^{88} \mathrm{Sr}^{+}$ ions using an oscillating electric field. The two ions, which were separated by just over $3.5 \mu \mathrm{m}$, were then laser cooled to $46 \mu \mathrm{K}$. The team then manipulated the quantum states of the ions with a laser, showing that they could couple these states to the ions'

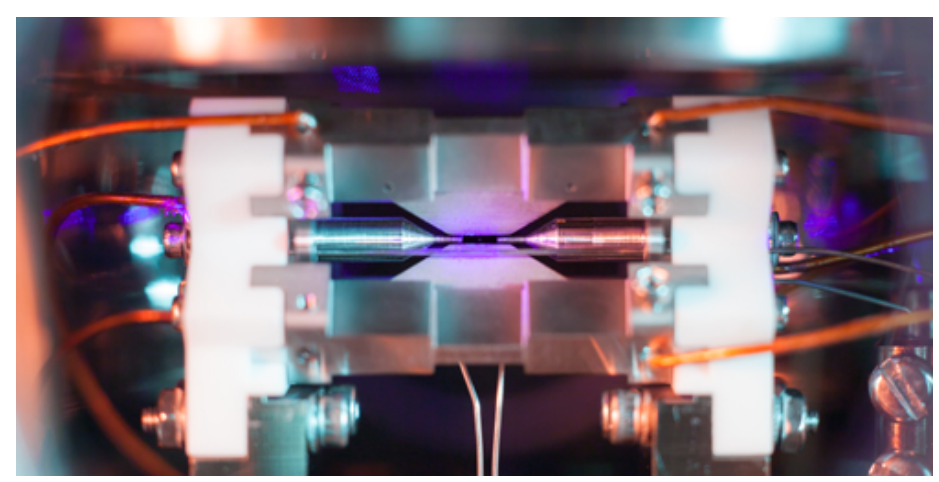

Credit: D. Nadlinger/University of Oxford shared motion and allow the ions to exchange information.

The team's approach is simpler than previous ones for entangling two different ions because the atomic transitions of ${ }^{43} \mathrm{Ca}^{+}$and ${ }^{88} \mathrm{Sr}^{+}$are close enough that both ions can be excited by a single laser. This simplification means that their setup has fewer beams to align and fewer calibration steps to enact, allowing the team to achieve a fidelity of well over $99 \%$ for their entanglement process-a necessary threshold for quantum error-correction processes. Such a simplified platform is also important for creating scalable quantum computing architectures.

Correction (18 August 2020): The story has been updated to correct the separation of the ions, which is $3.5 \mu \mathrm{m}$ and not $35 \mu \mathrm{m}$ as initially stated.

Katherine Wright is a Senior Editor for Physics.

\section{REFERENCES}

1. A. C. Hughes et al., "Benchmarking a high-fidelity mixed-species entangling gate,” Phys. Rev. Lett. 125, 080504 (2020). 